\title{
PERÍODO DE PERMANÊNCIA DE MUDAS DE Eucalyptus grandis EM VIVEIRO BASEADO EM PARÂMETROS MORFOLÓGICOS ${ }^{1}$
}

\author{
Eduardo Righi dos Reis², Alessandro Dal Col Lúcio³, Fabiano Oliveira Fortes ${ }^{4}$, Sidinei José Lopes ${ }^{3}$ e \\ Bruna Denardin da Silveira ${ }^{4}$
}

\begin{abstract}
RESUMO - Mesmo tendo avançado nas técnicas de produção de mudas, ainda existem muitos problemas a serem solucionados, principalmente no que se refere à expedição de mudas da casa de vegetação. Assim, este trabalho teve como objetivo verificar a variação espacial de mudas de Eucalyptus grandis ao longo do tempo, para estabelecer formas de expedição das mudas da casa de vegetação no viveiro florestal da Universidade Federal de Santa Maria, RS. Para tanto, foi instalado um experimento, em que se realizaram quatro avaliações com intervalo de 15 dias, e cada repetição foi dividida em parcelas para determinar a melhor forma de saída das mudas da casa de vegetação. Foi verificado maior homogeneidade entre as parcelas aos 100 dias após a emergência (DAE), dividindo-se a bandeja em Bordadura x Centro, Frente x Centro x Fundo e quadrantes e aos 115 DAE, para a simulação Direita x Centro x Esquerda, com a porção central apresentando sempre os melhores resultados. Houve variabilidade significativa entre posições dentro da bandeja e ao longo do tempo nas diferentes simulações de parcelas, e aos 100 DAE obteve-se a homogeneidade em todas as variáveis nas parcelas simuladas, como Frente x Centro x Fundo, podendo a porção central ser liberada aos 100 DAE, enquanto as demais porções tiveram de aguardar até os 115 DAE.
\end{abstract}

Palavras-chave: Expedição, uniformidade, variabilidade e Bartlett.

\section{PERIOD OF PERMANENCE OF Eucalyptus grandis SEEDLINGS IN NURSERY BASED ON MORPHOLOGICAL PARAMETERS}

\begin{abstract}
Even with the advance in seedling production techniques, many problems still remain to be solved, mainly in what refers to the expedition of seedlings from the vegetation house. This work aimed to verify the space variation of Eucalyptus grandis seedlings along time, to establish forms of expediting the seedlings from the vegetation house at the forest nursery of Santa Maria Federal University, RS. Thus, an experiment was set up consisting of four evaluations at 15 day intervals, with each repetition being divided into plots to determine the best form of expediting the seedlings from the vegetation house. A greater homogeneity was verified among the plots at 100 days after emergence (DAE), with the tray contents being divided into Border X Center, Front X Center X Bottom and quadrants and at 115 DAE for the Right X Center X Left simulation, with the central plot presenting the best results. A significant variability was verified among the plots within the tray, and along time for the different plot simulations. At 100 DAE, homogeneity was obtained in all the variables for the simulated plots, such as Front X Center X Bottom, with the central plot being released at 100 DAE, while the others only at 115 DAE.
\end{abstract}

Keywords: Expedition, uniformity, variability and Bartlett.

\footnotetext{
${ }^{1}$ Recebido em 11.02.2007 e aceito para publicação em 22.08.2008.

${ }^{2}$ Graduando em Química pela Universidade Federal de Santa Maria (UFSM).

${ }^{3}$ Departamento de Fitotecnia da UFSM. E-mail: <adlucio@smail.ufsm.br>.

${ }^{4}$ Programa de Pós-Graduação em Engenharia Florestal da UFSM. E-mail: <fabfortes@ yahoo.com.br>.
} 


\section{INTRODUÇÃO}

Os plantios florestais, em sua maioria, foram instalados com espécies de Pinus (P. elliottii e P. taeda) ou Eucalyptus (em sua maioria, E. saligna e E. grandis). Os plantios com Pinus concentram-se na Região Sul, totalizando $58 \%$ do total plantado. Essa distribuição se justifica pela alta adaptabilidade do gênero à região, fornecendo a base florestal para a indústria madeireira local. Os plantios com Eucalyptus concentram-se na Região Sudeste do Brasil, tendo o Estado de Minas Gerais $51 \%$ do total plantado com a espécie no país, seguido por São Paulo, com 19\% (ABIMCI, 2003).

A necessidade de produzir mudas em áreas bem definidas, com características específicas e controladas, deve-se ao fato de estas serem geralmente frágeis, necessitando de proteção inicial e de manejos especiais, obtendo, assim, maior uniformização de crescimento, tanto em altura quanto em sistema radicular. Quando as mudas atingem tamanho adequado, é suprimida a adubação e reduzida a irrigação, conduzindo à rustificação. Esse procedimento permite que as mudas resistam, após o plantio, às condições adversas encontradas a campo, assim como sua sobrevivência e crescimento satisfatórios, afirmaram Gomes et al. (2002).

A produção de mudas por meio de sementes utilizando tubetes de polipropileno acomodados em bandejas metálicas apresenta algumas vantagens, de acordo com Hoppe e Brun (2004), como a proteção do sistema radicular em toda a fase de desenvolvimento, a quantidade relativamente muito menor de substrato a ser utilizada, além de o enchimento dos recipientes ser um processo simples e de alto rendimento, pois estes são reutilizáveis, reduzem o espaço físico necessário à produção e apresentam ótima ergonomia de trabalho.

A altura das mudas na ocasião do transplante exerce importante papel na sobrevivência e desenvolvimento nos primeiros anos após essa operação. Há limites no crescimento em altura das mudas no viveiro, acima e abaixo das quais o desempenho não é satisfatório. Outro fator que tem que ser levado em consideração é o diâmetro do colo, de acordo com a espécie, o qual deve ser compatível com a altura para que seu desempenho no campo corresponda às expectativas (CARNEIRO, 1995).

Trabalhando com produção de mudas de diversas espécies florestais, Sturion et al. (2000) recomendaram a retirada de mudas de eucalipto da casa de vegetação quando estas atingirem em torno de 15 a $25 \mathrm{~cm}$ de altura e diâmetro de colo de 2,5 mm. De acordo com Gomes et al. (1996), as características nas quais as empresas florestais se fundamentam para a classificação das mudas de eucaliptos, na retirada destas da casa de vegetação, são baseadas nos parâmetros de altura média entre 15 e $30 \mathrm{~cm}$ e diâmetro do coleto de $2 \mathrm{~mm}$.

O plantio de mudas velhas tem sido um dos maiores problemas para o estabelecimento e o arranque inicial dos plantios no campo, segundo Mafia et al. (2005), pois, devido à permanência excessiva das mudas no viveiro, há redução do crescimento e enovelamento do sistema radicular. O aumento da porcentagem de sobrevivência decorre do uso de mudas de melhor padrão de qualidade, tornando, assim, dispensável o replantio, dada à pequena taxa de mortalidade que é verificada. Quando a sobrevivência não alcança índices aceitáveis, o replantio é necessário, assegurando maior aproveitamento aéreo e produção de madeira (CARNEIRO, 1995).

A relação altura/diâmetro do colo constitui um dos parâmetros usados para avaliar a qualidade de mudas florestais, de acordo com Sturion e Antunes (2000), pois, além de refletir o acúmulo de reservas, assegura maior resistência e melhor fixação no solo. Mudas com baixo diâmetro do colo apresentam dificuldades de se manterem eretas após o plantio, e o tombamento decorrente dessa característica pode resultar em morte ou deformações que comprometem o valor silvicultural dos indivíduos. Mudas que apresentam diâmetro do colo pequeno e alturas elevadas são consideradas de qualidade inferior às menores e com maior diâmetro do colo.

Há uma escassez de informações relacionadas às técnicas experimentais e desconhecimento de técnicas ou recursos estatísticos para experimentos em áreas restritas, conforme afirmativa de Lorentz et al. (2004), levando à obtenção de resultados imprecisos e recomendação de cultivares e tecnologias de maneira ineficiente. Para os referidos autores, as variações entre as diferentes avaliações, as linhas de cultivo e até mesmo entre as plantas são pouco estudadas e, muitas vezes, desconhecidas.

Assim, o objetivo deste trabalho foi verificar a variação espacial de mudas de Eucalyptus grandis ao longo do tempo, para estabelecer formas de expedição das mudas da casa de vegetação. 


\section{MATERIAL E MÉTODOS}

O experimento foi conduzido no viveiro florestal, em casa de vegetação, com dimensões de 6 × 30 m, pertencente ao Departamento de Ciências Florestais da Universidade Federal de Santa Maria, RS. As mudas de eucalipto (Eucalyptus grandis Hill ex-Maiden) foram semeadas em março de 2004, sendo constituído por duas repetições, compostas por 504 unidades básicas (UB) por repetição.

Os recipientes utilizados para a semeadura foram tubetes de polipropileno, modelo T53/4, com volume de $53 \mathrm{~cm}^{3}$, altura de $125 \mathrm{~mm}$, furo com diâmetro de 12 $\mathrm{mm}$, peso de $10 \mathrm{~g}$, sendo utilizado como substrato o Plantmax®. A irrigação foi realizada por uma barra de irrigação composta por aspersores do tipo microaspersão, com uma vazão de quatro $\mathrm{mm} / \mathrm{dia}$, que foi acionada por um timer no início da manhã às $7 \mathrm{~h}$ e a última às 18 h, sendo dividido em cinco irrigações diárias.

Para as simulações de expedição de mudas da casa de vegetação, dividiu-se cada uma das repetições em parcelas, de modo a avaliar o comportamento dos parâmetros morfológicos ao longo do tempo, definindo, assim, o período de expedição das mudas da casa de vegetação (Figura 1). As medições foram realizadas quinzenalmente, a partir dos 70 dias após a emergência (DAE), encerrando aos 115 DAE, avaliando a altura da parte aérea com régua graduada $(\mathrm{cm})$ e o diâmetro do colo com paquímetro digital $(\mathrm{cm})$. Em cada medição, em todas as simulações estimaram-se a média $(\bar{x})$ e o desvio-padrão (s) de cada uma das parcelas estudadas.

Utilizou-se o teste t para testar a significância das diferenças entre as médias de cada forma de parcela, dentro de cada data de avaliação. Em cada um dos períodos, aplicou-se o teste de homogeneidade das variâncias de Bartlett a 5\% de probabilidade de erro (STEEL et al., 1997), para verificar a uniformidade dos parâmetros morfológicos e relação altura/diâmetro (h/d).

\section{RESULTADOS E DISCUSSÃO}

Pelos valores do Qui-quadrado calculado, com respectivas significâncias pelo teste de Bartlett, nos diferentes períodos e nas diferentes formas de expedição de mudas: BXC, DXCXE, FrXCXF e quadrantes (Tabela 1), pode-se observar que, em algumas épocas de avaliação, houve heterogeneidade nos parâmetros morfológicos e relação h/d. Segundo Gomes et al. (2002), independentemente da idade, os parâmetros morfológicos de Eucalyptus grandis tendem a formar grupos homogêneos. Esse comportamento de heterogeneidade mostra que, com o crescimento e desenvolvimento das mudas, o comportamento destas não é uniforme, comprovando que há variabilidade significativa entre as plantas e entre as diferentes posições destas dentro da bandeja de produção.

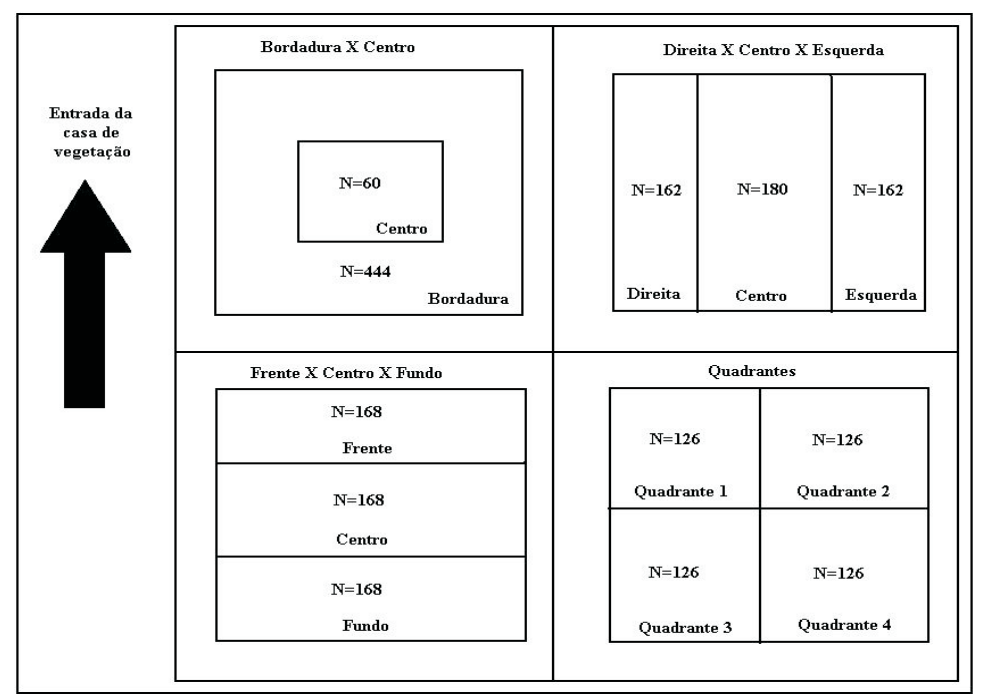

Figura 1 - Posições das diferentes simulações de parcelas para retiradas de mudas de Eucalyptus grandis produzidas em bandejas de 504 unidades básicas. Santa Maria, RS, 2006.

Figure 1 - Positions of the different plot simulations for translocation of the Eucalyptus grandis seedlings produced in 504 basic unit trays. Santa Maria, RS, 2006. 
Tabela 1 - Valores do $\chi^{2}$ calculado do teste de Bartlett nas diferentes simulações das variáveis diâmetro e altura e relação altura/diâmetro, avaliadas em quatro dias após a emergência (DAE). Santa Maria, RS, 2006

Table 1 - Values of the $\chi^{2}$ calculated by the Bartlett test for the different simulations of the variables diameter, height and height/diameter relationship, evaluated during four days after emergence(DAE). Santa Maria, RS, 2006

\begin{tabular}{|c|c|c|c|c|c|c|}
\hline \multirow[t]{2}{*}{ DAE } & \multicolumn{2}{|c|}{ Diâmetro $(\mathrm{cm})$} & \multicolumn{2}{|c|}{ Altura $(\mathrm{cm})$} & \multicolumn{2}{|c|}{ Relação h/d } \\
\hline & REP 1 & REP 2 & REP 1 & REP 2 & REP 1 & REP 2 \\
\hline & \multicolumn{6}{|c|}{ Bordadura X Centro } \\
\hline 70 & $5,36^{*}$ & $0,23^{\mathrm{NS}}$ & $0,10^{\mathrm{NS}}$ & $5,94 *$ & $8,46^{*}$ & $9,55^{*}$ \\
\hline 85 & $3,87 *$ & $0,00^{\mathrm{NS}}$ & 0,72 NS & $5,11 *$ & $20,48 *$ & $10,96^{*}$ \\
\hline 100 & $2,44^{\mathrm{NS}}$ & $0,08^{\mathrm{NS}}$ & $0,68^{\mathrm{NS}}$ & $3,80^{\mathrm{NS}}$ & $18,31 *$ & $15,09 *$ \\
\hline 115 & $1,74^{\mathrm{NS}}$ & 0,04 Ns & $1,06^{\mathrm{NS}}$ & $4,64 *$ & $4,92 *$ & $100,17 *$ \\
\hline$\chi_{\text {Tabelado }}^{2}$ & \multicolumn{6}{|c|}{3,84} \\
\hline & \multicolumn{6}{|c|}{ Direita X Centro X Esquerda } \\
\hline 70 & $29,16^{*}$ & $4,72^{\mathrm{NS}}$ & 0,39 NS & $2,49^{\mathrm{NS}}$ & $0,54^{\mathrm{NS}}$ & $23,63 *$ \\
\hline 85 & $2,29^{\mathrm{NS}}$ & $5,43^{\mathrm{NS}}$ & 3,25 NS & $1,26^{\mathrm{NS}}$ & $13,50 *$ & $0,61^{\mathrm{NS}}$ \\
\hline 100 & $3,41 \mathrm{NS}$ & $6,72 *$ & 5,15 NS & $0,42^{\mathrm{NS}}$ & $14,64 *$ & $2,36^{\mathrm{NS}}$ \\
\hline 115 & 1,71 Ns & $1,13^{\mathrm{NS}}$ & 3,84 Ns & $0,16^{\mathrm{NS}}$ & $7,08 *$ & $366,88 *$ \\
\hline$\overline{\chi_{\text {Tabelado }}^{2}}$ & \multicolumn{6}{|c|}{5,99} \\
\hline & \multicolumn{6}{|c|}{ Frente X Centro X Fundo } \\
\hline 70 & $23,58 *$ & $5,81^{\mathrm{NS}}$ & 3,81 Ns & $2,54 \mathrm{NS}$ & $3,15^{\mathrm{NS}}$ & $0,26^{\mathrm{NS}}$ \\
\hline 85 & $1,17^{\mathrm{NS}}$ & $0,76^{\mathrm{NS}}$ & $3,28^{\mathrm{NS}}$ & $2,93^{\mathrm{NS}}$ & $7,31 *$ & $1,86^{\mathrm{NS}}$ \\
\hline 100 & $0,41^{\mathrm{NS}}$ & $1,05^{\mathrm{NS}}$ & $1,85^{\mathrm{NS}}$ & $4,20^{\mathrm{NS}}$ & $3,54^{\mathrm{NS}}$ & $1,23^{\mathrm{NS}}$ \\
\hline 115 & 0,29 Ns & $1,11^{\mathrm{NS}}$ & $2,19^{\mathrm{NS}}$ & $6,68 *$ & $4,27^{\mathrm{NS}}$ & $321,96 *$ \\
\hline$\chi_{\text {Tabelado }}^{2}$ & \multicolumn{6}{|c|}{5,99} \\
\hline & \multicolumn{6}{|c|}{ Quadrantes } \\
\hline 70 & $36,90 *$ & 1,42 Ns & $2,58^{\mathrm{NS}}$ & $0,16^{\mathrm{NS}}$ & $3,44^{\mathrm{NS}}$ & $0,89^{\mathrm{NS}}$ \\
\hline 85 & $1,74^{\mathrm{NS}}$ & $0,27^{\mathrm{NS}}$ & $5,21^{\mathrm{NS}}$ & $0,25^{\mathrm{NS}}$ & $10,02 *$ & $5,40^{\mathrm{NS}}$ \\
\hline 100 & $2,38^{\mathrm{NS}}$ & $1,21^{\mathrm{NS}}$ & $3,57 \mathrm{Ns}$ & $1,02^{\mathrm{NS}}$ & $5,24^{\mathrm{NS}}$ & $4,61^{\mathrm{NS}}$ \\
\hline 115 & $0,81^{\mathrm{NS}}$ & $0,78^{\mathrm{NS}}$ & $2,88^{\mathrm{NS}}$ & $1,04^{\mathrm{NS}}$ & $1,72^{\mathrm{NS}}$ & $385,44 *$ \\
\hline$\overline{\chi_{\text {Tabelado }}^{2}}$ & \multicolumn{6}{|c|}{7,81} \\
\hline
\end{tabular}

NS variâncias homogêneas $(\mathrm{p}>0,05)$; variâncias heterogêneas $(\mathrm{p} \leq 0,05)$.

Considerando a relação entre os parâmetros morfológicos, para avaliar a qualidade das mudas verificouse grande proporção de homogeneidade aos 100 DAE, representando, assim, a uniformidade dos parâmetros diâmetro do coleto e altura, exceto na simulação DXCXE (Tabela 1). Gomes et al. (2002), avaliando os parâmetros morfológicos na qualidade de mudas de Eucalyptus grandis, concluíram que aos 90 dias de idade estas apresentavam crescimento adequado para o plantio, e após 90 dias de idade os volumes dos tubetes começam a restringir o crescimento das mudas. Os mesmos autores recomendaram ainda a não-retirada das mudas aos 60 dias, pois elas ainda estão pequenas e bastante tenras, não se adequando para o plantio a campo. Eles não recomendam a retirada das mudas com mais de 120 dias após a semeadura, pois as mudas aumentam o diâmetro do coleto e ganham mais peso de matéria seca, apresentando altura muito superior à ideal para o plantio. Esse resultado vem confirmar a homogeneidade observada nas mudas aos 100 DAE, indicando que para três simulações com variâncias homogêneas esse período é o adequado para a liberação das mudas como mesmo padrão de qualidade. Já na simulação DXCXE a maior proporção de homogeneidade foi apresentada aos 115 DAE (Tabela 1). Dessa forma, as comparações entre as diferentes parcelas simuladas foram realizadas nessas épocas, quando houve comportamento de homogeneidade, pois se entende que nesse ponto as mudas estão em condições similares de crescimento e desenvolvimento.

Nos resultados apresentados pelas médias dos parâmetros morfológicos, nas diferentes simulações de parcelas verificou-se que a altura mínima recomendada, para a expedição de mudas de Eucalyptus grandis, foi atingida aos 100 DAE (Tabela 2), conforme descreveram Gomes et al. (1996) e Sturion et al. (2000), e, na maioria das avaliações, independentemente da 
forma de retirada das mudas, houve diferenças significativas entre as parcelas testadas. Já na variável diâmetro do coleto a recomendação mínima, descrita pelos referidos autores, não foi atendida na maioria das situações simuladas (Tabela 2).

O teste $\mathrm{t}$, entre as médias dos parâmetros morfológicos, mostrou contrastes significativos para diâmetro, altura e relação h/d, entre a bordadura e o centro da bandeja (Tabela 2). Dessa forma, a retirada das mudas da bandeja aos 100 DAE deve ser realizada de maneira escalonada, favorecendo a saída da porção central, de modo a obter na retirada mudas com um mesmo padrão de diâmetro e altura.

Observou-se que, durante as avaliações, a bordadura das repetições sofreu maior influência das condições ambientais da casa de vegetação, fazendo que o substrato dos recipientes ressecasse, influenciando o tamanho das mudas. Ferreira et al. (1999), estudando a relação hídrica em mudas de Eucalyptus citriodora produzidas em tubetes, afirmaram que o potencial foliar refletia as condições da dinâmica do processo de transporte no sistema solo-planta-atmosfera, constituindo o principal componente responsável pelo fluxo de água na planta. De acordo com esses mesmos autores, o déficit de água na bordadura ocasiona uma diferença na resposta estomática ao estresse, interferindo na capacidade de produção de ácido abscísico e inibindo, assim, o desenvolvimento das plantas nessa região.

A comparação dos diferentes métodos de retiradas das mudas da casa de vegetação obtidas neste experimento, visando à formação de mudas com características fenotípicas superiores para Eucalyptus grandis em determinado período, indica que aos 100 DAE, utilizando o sistema de expedição de mudas FrXCXF, todos os parâmetros morfológicos se apresentaram uniformes (Tabela 1). Entretanto, os valores recomendados para expedição das mudas foram obtidos com superioridade significativa na parte central (Tabela 2), mesmo comportamento observado nos demais tipos de simulações nos quais houve porção central.

Num processo maior de refinamento, selecionamse as mudas que apresentem uniformidade entre seus parâmetros morfológicos que permitiram ganhos excepcionais em produtividade. Dessa forma, há uma recomendação, independentemente do modo de retirada das mudas, de que a porção central deve ser liberada em um primeiro momento, ficando por mais tempo no viveiro as mudas das demais porções da bandeja.

Tabela 2 - Média do diâmetro do coleto (d) e da altura da parte aérea (h) e relação altura/diâmetro (h/d), em mudas de Eucalyptus grandis em parcelas simuladas Bordadura x Centro, Frente x Centro X Fundo e quadrantes, avaliadas aos 100 dias após a emergência, e Direita x Centro x Esquerda, avaliadas aos 115 dias após a emergência. Santa Maria, RS, 2006

Table 2 - Diameter mean $(d)$, height of the aerial part ( $h$ ), and height/diameter relationship $(h / d)$, in Eucalyptus grandis seedlings in simulated plots Border X Center, Front X Center X Bottom and quadrants, evaluated at 100 days after emergence and Right $x$ Center $x$ Left, evaluated at 115 days after emergence. Santa Maria, RS, 2006

\begin{tabular}{|c|c|c|c|c|c|c|}
\hline \multirow[t]{2}{*}{ Parcelas simuladas } & \multicolumn{3}{|c|}{ Repetição 1} & \multicolumn{3}{|c|}{ Repetição 2} \\
\hline & $\mathrm{d}(\mathrm{cm})$ & $\mathrm{h}(\mathrm{cm})$ & Relação h/d & $\mathrm{d}(\mathrm{cm})$ & $\mathrm{h}(\mathrm{cm})$ & Relação h/d \\
\hline Bordadura & $1,69 b^{*}$ & $15,10 b$ & $8,71 b$ & $1,72 b$ & $15,18 b$ & $8,68 b$ \\
\hline Centro & $1,95 \mathrm{a}$ & $20,03 \mathrm{a}$ & $10,24 a$ & $1,89 \mathrm{a}$ & $19,65 a$ & $10,44 \mathrm{a}$ \\
\hline Frente & $1,65 b$ & $13,70 \mathrm{c}$ & $8,06 \mathrm{c}$ & $1,83 \mathrm{a}$ & $17,36 \mathrm{a}$ & $9,45 \mathrm{a}$ \\
\hline Centro & $1,80 \mathrm{a}$ & $18,01 \mathrm{a}$ & $9,85 \mathrm{a}$ & $1,76 \mathrm{a}$ & $16,69 a$ & $9,29 \mathrm{a}$ \\
\hline Fundo & $1,71 \mathrm{ab}$ & $15,26 \mathrm{~b}$ & $8,75 b$ & $1,62 b$ & $13,13 b$ & $7,95 b$ \\
\hline Quadrante1 & $1,70 \mathrm{a}$ & $15,90 \mathrm{bc}$ & $8,98 b$ & $1,79 \mathrm{a}$ & $17,27 \mathrm{a}$ & $9,51 \mathrm{a}$ \\
\hline Quadrante2 & $1,79 \mathrm{a}$ & $17,78 \mathrm{a}$ & $9,75 \mathrm{a}$ & $1,72 \mathrm{ab}$ & $15,39 b$ & $8,81 \mathrm{~b}$ \\
\hline Quadrante3 & $1,66 \mathrm{a}$ & $13,94 \mathrm{c}$ & $8,12 \mathrm{c}$ & $1,82 \mathrm{a}$ & $17,04 \mathrm{a}$ & $9,38 \mathrm{ab}$ \\
\hline Quadrante4 & $1,72 \mathrm{a}$ & $14,89 \mathrm{bc}$ & $8,64 b c$ & $1,62 b$ & $13,25 c$ & $7,90 \mathrm{c}$ \\
\hline Direita & $1,89 b$ & $18,77 b$ & $9,69 \mathrm{a}$ & $1,94 b$ & $17,08 \mathrm{~b}$ & $8,67 b$ \\
\hline Centro & $2,04 \mathrm{a}$ & $20,30 a$ & $9,88 \mathrm{a}$ & $2,08 \mathrm{a}$ & $20,05 a$ & $9,60 \mathrm{a}$ \\
\hline Esquerda & $1,83 b$ & $14,58 \mathrm{c}$ & $7,76 b$ & $1,85 b$ & $14,78 \mathrm{c}$ & $8,20 b$ \\
\hline
\end{tabular}

* Letras diferentes em cada coluna, dentro de cada simulação, diferem pelo peste t $(\mathrm{p}>0,05)$. 


\section{CONCLUSÕES}

Houve variabilidade significativa entre posições dentro da bandeja e ao longo do tempo nas diferentes simulações de parcelas.

Aos 100 dias após a emergência (DAE), obtevese a homogeneidade em todas as variáveis das parcelas simuladas como Frente X Centro X Fundo.

Para um melhor padrão na liberação de mudas de Eucalyptus grandis, a porção central deverá ser liberada aos 100 DAE, enquanto as demais porções deverão permanecer até os 115 DAE.

\section{REFERÊNCIAS}

ASSOCIAÇÃO BRASILEIRA DA INDÚSTRIA DE MADEIRA PROCESSADA MECANICAMENTE ABIMCI. 2003. Estudo Setorial 2003 Produtos de Madeira Sólida. Disponível em: <http://www.abimci.com.br/port/03Dados/ 0306EstSet2003/0306Quadro.html>. Acesso em: 14 de fev. 2006.

CARneiro, J. G. A. Produção e controle de qualidade de mudas florestais. Curitiba: UFPR/FUPEF, 1995. 451p.

FERREIRA, C.A. G.; DAVIDE, A. C.; CARVALHO, L. R. Relações hídricas em mudas de Eucalyptus citriodora Hook., em tubetes, aclimatadas por tratamentos hídricos. Cerne, v.5, n.2, p.95-104, 1999.

GOMES, J. M., PAIVA, H. N.; COUTO, L. Produção de mudas de eucalipto. Informe Agropecuário, v.18, n.185, p.15-23, 1996.
GOMES, J. M. et al. Parâmetros morfológicos na avaliação da qualidade de mudas de Eucalyptus grandis, Revista Árvore, v.26, n.6, p.655-664, 2002.

HOPPE, J. M.; BRUN, E. J. Produção de sementes e mudas florestais. Santa Maria: 2004. 125p. (Caderno Didático)

LORENTZ, L. H. et al. Distribuição da variabilidade produtiva do pimentão em cultivo protegido. Horticultura Brasileira, v.22, n.2, 2004. CD-ROM. Suplemento

MAFIA, R. G. et al. Critério técnico para determinação da idade ótima de mudas de eucalipto para plantio. Revista Árvore, v.29, n.6, p.947-953, 2005.

STEEL, R. G. D.; TORRIE, J. H.; DICKEY, D. A. Principles and procedures of statistics: a biometrical approach. New York: McGraw-Hill, 1997. 666p.

STURION, J. A.; GRAÇÃ, L. R.; ANTUNES, J. B. $M$. Produção de mudas de espécies de rápido crescimento por pequenos produtores. Colombo: Embrapa Florestas, 2000. 20p. (Circular Técnica, 37)

STURION, J. A.; ANTUNES, B. M. A. Produção de mudas de espécies florestais. In: GALVÃO, A.P.M.

Reflorestamento de propriedades rurais para fins de produtivos e ambientais. Colombo: 2000. p.125-150. 